\title{
Antibody-secreting macrophages generated using CpG-free plasmid eliminate tumor cells through antibody-dependent cellular phagocytosis
}

\author{
Eun Bi Cha ${ }^{1,2}$, Keun Koo Shin ${ }^{1}$, Jinho Seo ${ }^{1, *}$ E Doo-Byoung $\mathrm{Oh}^{1,2, *}$ \\ ${ }^{1}$ Environmental Disease Research Center, Korea Research Institute of Bioscience and Biotechnology (KRIBB), Daejeon 34141 , ${ }^{2}$ Department \\ of Biosystems and Bioengineering, KRIBB School of Biotechnology, University of Science and Technology (UST), Daejeon 34113, Korea
}

\begin{abstract}
The non-viral delivery of genes into macrophages, known as hard-to-transfect cells, is a challenge. In this study, the microporation of a CpG-free and small plasmid (pCGfd-GFP) showed high transfection efficiency, sustainable transgene expression, and good cell viability in the transfections of Raw 264.7 and primary bone marrow-derived macrophages. The non-viral method using the pCGfd vector encoding anti-EGFR single-chain Fv fused with Fc (scFv-Fc) generated the macrophages secreting anti-EGFR scFv-Fc. These macrophages effectively phagocytized tumor cells expressing EGFR through the antibody-dependent mechanism, as was proved by experiments using EGFR-knockout tumor cells. Finally, peri-tumoral injections of anti-EGFR scFv-Fc-secreting macrophages were shown to inhibit tumor growth in the xenograft mouse model. [BMB Reports 2020; 53(8): 442-447]
\end{abstract}

\section{INTRODUCTION}

Immune cell therapy has been highlighted owing to the success of the engineered T cells expressing chimeric antigen receptors (CARs) (1). Although CAR T cell (CAR-T) therapy has been used to treat B-cell-derived lymphoma, the application of CAR-T against solid tumors has not been successful. To solve this problem, alternative immune cells, such as natural killer cells and macrophages, have attracted attention (1). Macrophages are professional phagocytes that engulf and digest pathogenic microbes, dead cells, and cellular debris. It has been reported that the phagocytic ability of macrophages plays an important role in anti-cancer

*Corresponding authors. Doo-Byoung Oh, Tel: +82-42-860-4457; Fax: +82-42-879-8299; E-mail: dboh@kribb.re.kr; Jinho Seo, Tel: +8242-879-8296; Fax: +82-42-879-8299; E-mail: sjh0130@kribb.re.kr

https://doi.org/10.5483/BMBRep.2020.53.8.024

Received 5 February 2020, Revised 22 February 2020, Accepted 28 February 2020

Keywords: Antibody-dependent cellular phagocytosis, Antibody-secreting macrophage, CpG-free plasmid, Macrophages, Non-viral gene delivery therapy, using monoclonal antibodies, such as rituximab, trastuzumab, daratumumab, and elotuzumab (2-5). The antibody binding to tumor-specific antigens results in an antibody coating of the tumor cell surfaces, which is known as opsonization. Then, macrophages efficiently engulf antibody-opsonized tumor cells via antibody-dependent cellular phagocytosis (ADCP) (6).

Initial clinical trials using autologous macrophages did not result in any meaningful therapeutic effects on cancer treatment, because the tumor microenvironment can polarize macrophages into the pro-tumorigenic phenotype (7). Therefore, pre-treatments, such as by ex vivo genetic engineering, are required for the macrophages to eliminate tumor cells (8). For the genetic engineering of macrophages, viral gene-delivery methods have been widely used because of their high transfection efficiency and long-term expression. However, their clinical applications are highly limited because of various potential concerns, including oncogenic transformation, pathogenic risks, and immune responses $(9,10)$. Many efforts have been made to develop clinically safe non-viral gene-delivery systems using plasmid vectors, which have the advantage of easy production as well as a lower possibility of chromosome integration. However, these strategies suffer from low transfection efficiency and short sustainability; moreover, macrophages are known as "hard-to-transfect" cells (11). Many strategies for the engineering of plasmids have been developed to increase transfection efficiency (12). A decreased plasmid size is one of the factors known to improve transfection efficiency, indicating that shortness is a crucial factor for vector design; this has led to the development of minimized vectors, such as minicircles and MIDGE $(12,13)$. Manipulating vector components have also been shown to increase transgene expression (12). The plasmids produced from Escherichia coli generally contain unmethylated cytosine-phosphate-guanine (CpG) dinucleotide sequences, which are recognized by the mammalian immune system through Toll-like receptor 9 (TLR9) and are known to induce both inflammatory responses and transgene silencing (14). Plasmids devoid of CpG sequences have been previously developed and used to improve transgene expression in the various tissues (15-21).

In this study, we employed a non-viral gene-delivery method using a CpG-free plasmid for the generation of macrophages 
that secrete anti-EGFR antibody. They efficiently eliminated tumor cells expressing EGFR through ADCP. The peri-tumoral injection of antibody-secreting macrophages suppressed tumor growth in a xenograft mouse model, indicating their potential use in the development of immune cell therapies.

\section{RESULTS}

\section{Plasmids lacking $\mathrm{CpG}$ sequences greatly improved the transfection efficiency of macrophages}

Although plasmids devoid of $\mathrm{CpG}$ sequences have been demonstrated to have high rates of transfection in several types of tissues and cell lines (15-21), there are currently no reports on their effect on macrophage transfection. To determine whether the removal of $\mathrm{CpG}$ sequences from plasmids improves transfection efficiency in macrophages, we used a commercially available CpG-free plasmid, pCpGfree-Lucia (3.6 kb), as a plasmid backbone. To facilitate this measurement, the reporter gene expressing Lucia luciferase was replaced with the GFP gene, resulting in the pCGf-GFP plasmid $(3.7 \mathrm{~kb})$ (Supplementary Fig. 1). Because smaller plasmids are associated with a better transfection efficiency $(12,13)$, we removed two MARs (IFN- $\beta$ S/MAR and $\beta$-globin MAR) from the pCGf-GFP plasmid, creating pCGfd-GFP (2.5 kb) (Supplementary Fig. 1). As a control plasmid, pcDNA3.1 expressing GFP (pcDNA3.1-GFP) was also constructed.

Microporation was used to deliver the plasmids into the RAW 264.7 macrophages. The microporation of the pCGf-GFP and pCGfd-GFP plasmids generated a much larger population of GFP-positive $\left(\mathrm{GFP}^{+}\right)$cells than that of pcDNA3.1-GFP (Fig. $1 \mathrm{~A})$. We analyzed the percentages of the $\mathrm{GFP}^{+}$cells for pCGfGFP and pCGfd-GFP by flow cytometry and found them to be
$70 \%$ and $85 \%$, respectively, whereas pcDNA3.1-GFP displayed a percentage of only $22 \%$ (Fig. 1B). Notably, the smallest plasmid, pCGfd-GFP, provided the highest efficiency. When the sustainability of GFP expression was analyzed for six days, the pCGfGFP and pCGfd-GFP microporations exhibited a longer GFP expression than did pcDNA3.1-GFP (Fig. 1C). The fact that the pCGfd-GFP plasmid displayed sustainability like that of pCGf-GFP indicated that the beneficial effect of plasmid size reduction was greater than the negative effect induced by the removal of two MARs.

Because unmethylated $\mathrm{CpG}$ sequences have been known to induce inflammatory responses and cell cytotoxicity (15), next, we determined whether a reduction in $\mathrm{CpG}$ sequences increases cell viability. When the viabilities were analyzed one day after the microporation (Fig. 1D), pCGf-GFP and pCGfd-GFP showed significantly higher viabilities (56\% and $66 \%$ ) than did pcDNA3.1GFP $(35 \%)$.

Based on the results of the transfection efficiency, sustainability, and viability experiments, we selected pCGfd-GFP as the best plasmid for RAW 264.7 macrophage transfection.

\section{pCGfd-GFP showed the best performance in primary macrophage transfection}

Next, we investigated the transfection of primary BMDMs because it is known to be extremely challenging (11). After testing a series of microporation conditions, we found an optimal condition (10 $\mu$ g of plasmid, $1680 \mathrm{~V}, 20 \mathrm{~ms}$, and one pulse) (Fig. 2A). More plasmid was required for efficient BMDM transfection than for RAW 264.7 macrophage transfection. Similar to the results for RAW 264.7 macrophage transfection, pCGfd-GFP showed the highest percentage $(\sim 50 \%)$ of $\mathrm{GFP}^{+}$cells and the
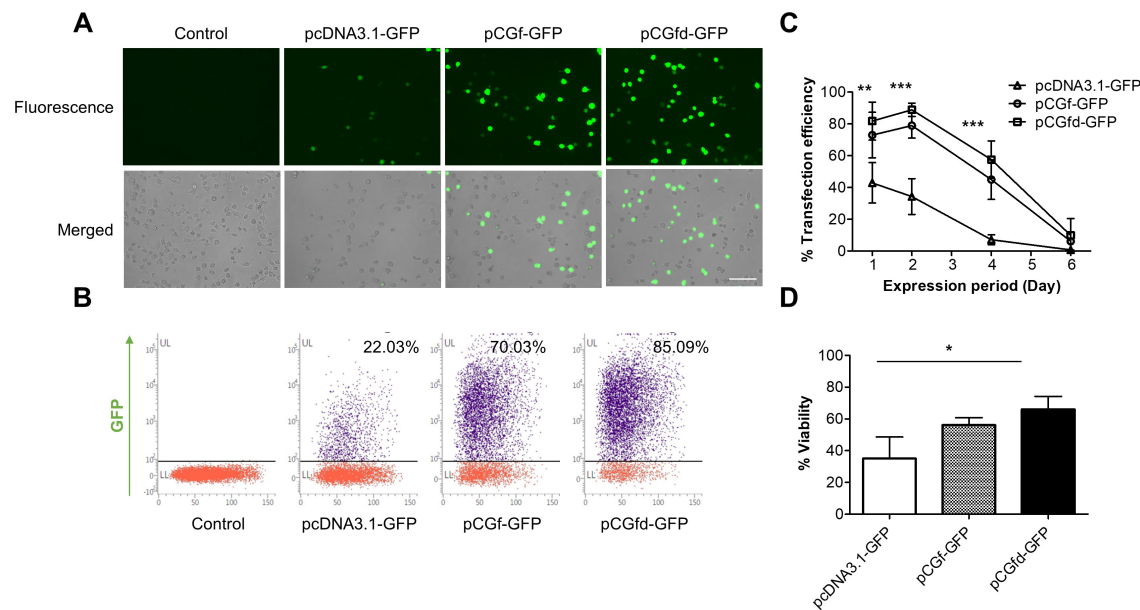

Fig. 1. Improved transfection efficiency, prolonged sustainability, and increased cell viability by plasmids lacking CpG sequences. (A) GFP ${ }^{+}$ RAW 264.7 macrophages were analyzed by fluorescence microscopy two days after microporation. Scale bar $=100 \mu$ m. (B) GFP ${ }^{+}$macrophages were analyzed by flow cytometry. (C) The percentages of GFP ${ }^{+}$macrophages were quantified for six days by flow cytometry. (D) Cell viability was measured using a Luna Dual fluorescence cell counter. Data represent the means \pm standard deviation (SD), $\mathrm{n}=3$. Statistical significances were determined by two-way $(C)$ or one-way (D) ANOVA, $* P<0.05, * * P<0.01, * * * P<0.001$. 

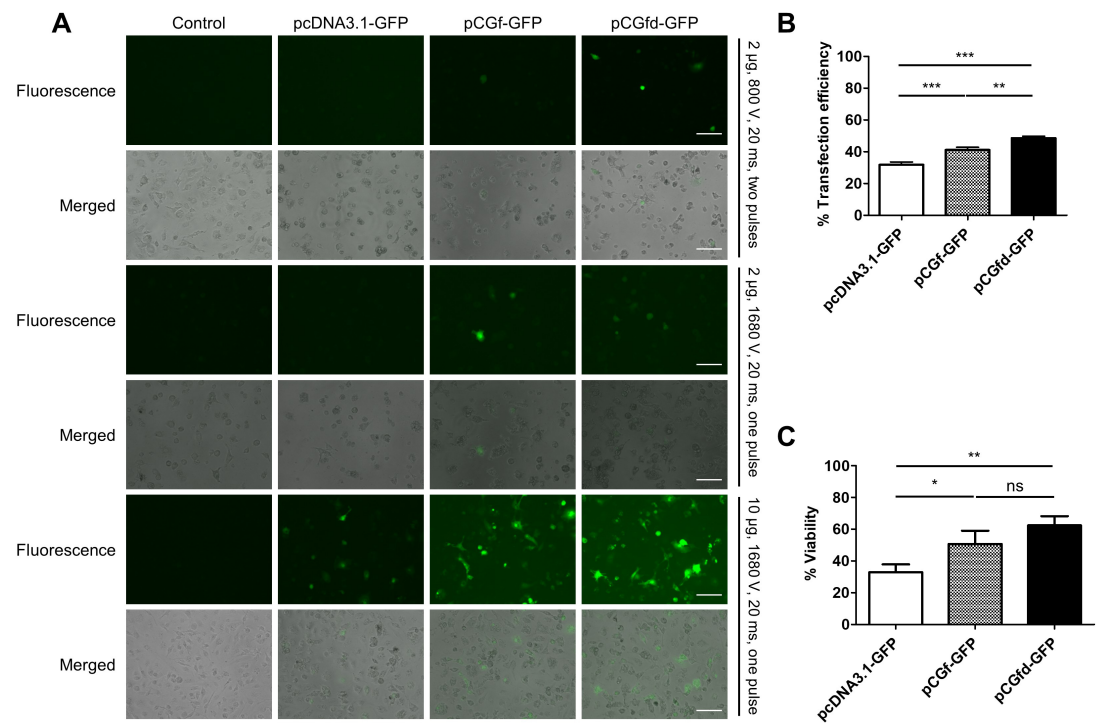

Fig. 2. Efficient transfection and good cell viability in BMDMs by pCGfd-GFP microporation. (A) GFP ${ }^{+}$BMDMs were analyzed by fluorescence microscopy two days after microporation. Scale bar $=100 \mu \mathrm{m}$. (B) The percentages of GFP ${ }^{+}$BMDMs were analyzed by flow cytometry. (C) Cell viability was measured using a Luna Dual fluorescence cell counter. Data represent the means \pm SD, $\mathrm{n}=3$. Statistical significances were determined by one-way ANOVA, $* P<0.05, * * P<0.01, * * *<0.001$.

maximum cell viability upon BMDM transfection (Fig. 2B and 2C).

\section{Antibody-secreting macrophages can efficiently remove tumor cells through ADCP}

We designed the pCGfd vector for the secretion of anti-EGFR scFv fused with Fc (anti-EGFR scFv-Fc), because macrophages can recognize the $\mathrm{FC}$ region of antibody-opsonized tumor cells for ADCP. To this end, the pCGfd-aEGFR-scFv-Fc encoding the signal peptide, anti-EGFR scFv, and Fc of IgG were constructed (Supplementary Fig. 2). Here, the anti-EGFR scFv sequences were derived from the light- and heavy-chain variable regions of cetuximab, which is currently used for anti-cancer therapy. After RAW 264.7 macrophage transfection, the amounts of antiEGFR scFv-Fc gradually increased in the culture media for three days (Fig. 3A).

The analysis of phagocytosis was performed using three tumor cell lines, A431, HT-29, and A673, representing high-, medium-, and low-EGFR-expressing cells, respectively (Supplementary Fig. 3). The anti-EGFR scFv-Fc-secreting RAW 264.7 macrophages were found to efficiently phagocytize A431 and HT-29 cells (Fig. 3B and 3C), but the control macrophages did not. We used flow cytometry for the quantitative analysis of phagocytosis after co-incubating the macrophages and tumor cells for $2 \mathrm{~h}$ at various ratios (1:0.1, 1:0.2, 1:1, and 1:2) (Fig. 3E3G). The anti-EGFR scFv-Fc-secreting macrophages showed a 3- to 7-fold higher percentage of phagocytosis (\%) of A431 and HT-29 cells than did the control macrophages (Fig. 3E and 3F). The A673 cells expressing low levels of EGFR were also engulfed by the anti-EGFR scFv-Fc-secreting macrophages, although the phagocytosis was much less efficient (Fig. 3G).

To confirm that phagocytosis occurred by binding anti-EGFR scFv-Fc to EGFR on the surface of the tumor cells, we constructed EGFR $^{\mathrm{KO}}$ A431 cells using the CRISPR-Cas9 system based on the pCGfd vector (pCGfd-gR-EGFR-Cas9-2A-GFP) for efficient transfection (Supplementary Fig. 4). As expected, antiEGFR scFv-Fc-secreting macrophages phagocytized very few EGFR $^{K O}$ A431 cells, which was in contrast with the efficient phagocytosis of wild-type A431 cells (Fig. $3 \mathrm{H}$ and 3I). These results clearly indicate that anti-EGFR scFv-Fc macrophages phagocytize EGFR-expressing tumor cells via ADCP.

\section{Anti-EGFR scFv-Fc-secreting macrophages inhibit tumor growth in vivo}

Last, we investigated whether anti-EGFR scFv-Fc-secreting macrophages were able to remove EGFR-expressing tumor cells in vivo using a xenograft mouse experiment (Fig. 4A). The peritumoral injection of anti-EGFR scFv-Fc-secreting RAW 264.7 macrophages clearly slowed A431 tumor growth more than did the injection of the control or the macrophages (Fig. 4B and $4 \mathrm{C}$ ). Furthermore, the tumor volumes and masses were significantly smaller only in the mice injected with anti-EGFR scFv-Fc-secreting macrophages (Fig. 4D and 4E). No differences were found between the PBS and macrophage injections, which strongly suggests that the genetic engineering of macrophages for the secretion of antibodies is essential for anti-tumor therapy. 

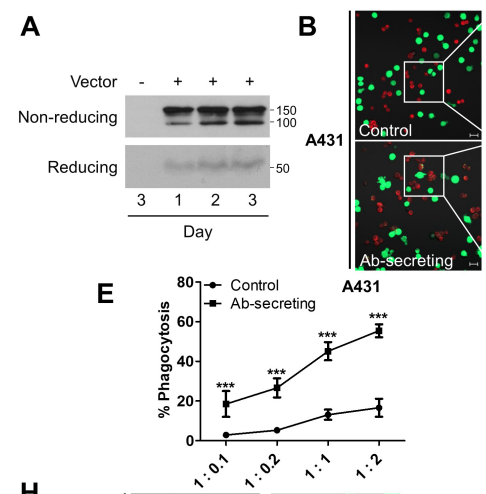

H
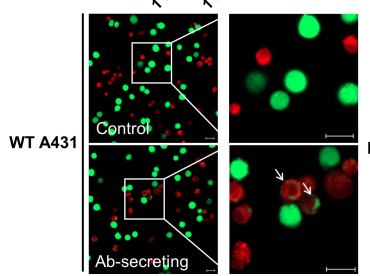
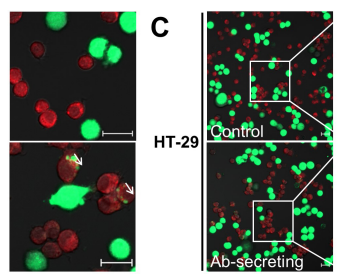

$\mathbf{F}$
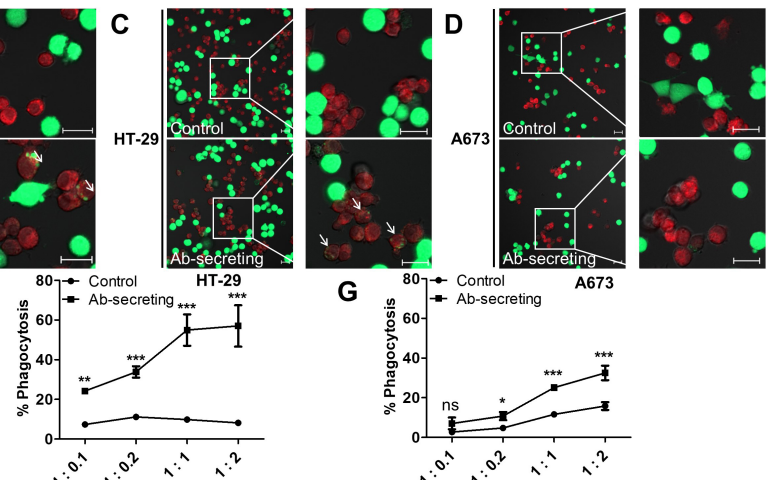

G
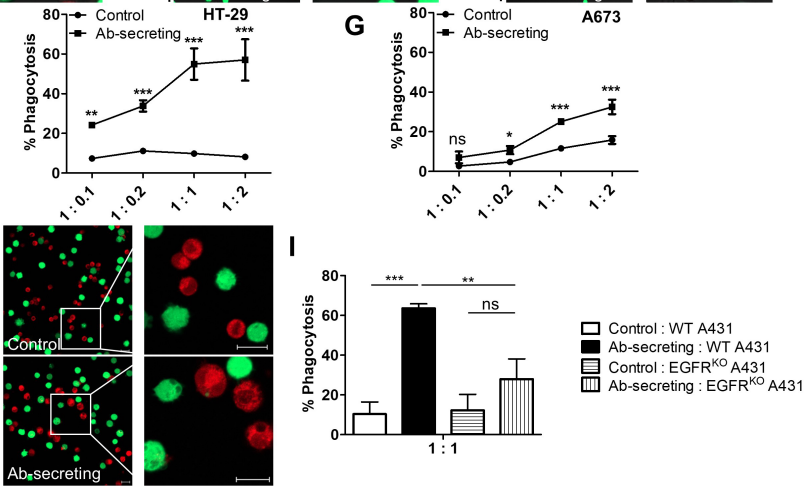

Fig. 3. Anti-EGFR scFv-Fc-secreting macrophages efficiently phagocytized tumor cells expressing EGFR. (A) After pCGfd-aEGFR-scFv-Fc microporation, the culture media of the RAW 264.7 macrophages were collected for three days and analyzed in non-reducing and reducing conditions by immunoblotting using anti-human Fc antibody. (B-D) Deep red dye-stained macrophages (control and Ab-secreting) were co-incubated for $2 \mathrm{~h}$ with Calcein-stained (green fluorescent) A431 (B), HT-29 (C), or A673 (D) cells. The arrows indicate the macrophages phagocytizing the tumor cells. Scale bar $=20 \mu \mathrm{m}$. (E-G) After $2 \mathrm{~h}$ of co-incubating the macrophages and A431 (E), HT-29 (F), or A673 $(\mathrm{G})$, the cells were analyzed by flow cytometry. The ratios of macrophage versus tumor cells were 1:0.1, 1:0.2, 1:1, and 1:2. ( $\mathrm{H}$, I) After co-incubating the macrophages with the WT or EGFR ${ }^{\mathrm{KO}}$ A431 cells, phagocytosis was analyzed by confocal microscopy $(\mathrm{H})$ and flow cytometry (I). Scale bar $=20 \mu \mathrm{m}$. Data represent the means $\pm \mathrm{SD}, \mathrm{n}=3$. Statistical significances were determined by using two-way (E-G) or one-way (I) ANOVA, ns $=$ non-significance, ${ }^{*} \mathrm{P}<0.05,{ }^{* *} \mathrm{P}<0.01,{ }^{* * *} \mathrm{P}<0.001$.

A

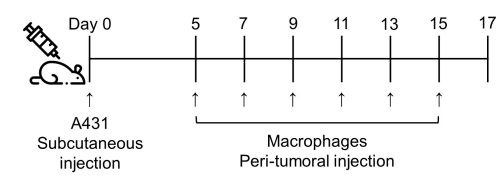

C

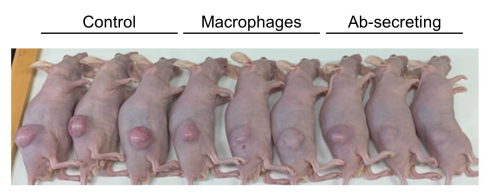

D
B

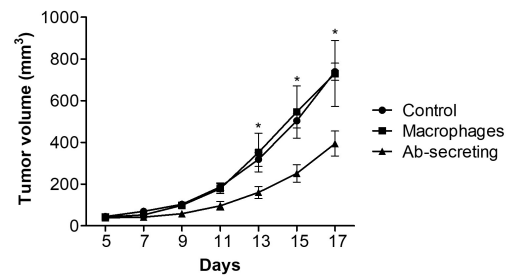

E

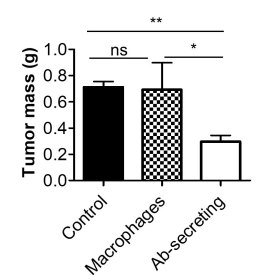

Fig. 4. Anti-EGFR scFv-Fc-secreting macrophages effectively inhibited tumor growth in vivo. (A) Schematic representation of the xenograft experiment. (B) The tumor volumes were measured on the indicated days. (C-E) Photos of the mice (C) and the incised tumors (D) were obtained after sacrifice. The tumor masses were measured (E). Data represent the means \pm standard error mean $(\mathrm{SEM})$, $\mathrm{n}=5$. Statistical significance was determined by using the one-tailed Mann Whitney U-test, $\mathrm{ns}=$ non-significance, ${ }^{*} \mathrm{P}<0.05,{ }^{* *} \mathrm{P}<0.01$.

\section{DISCUSSION}

The potential of genetically engineering immune cells has been apparent, because CAR-T was used to achieve a successful cure for B-cell-derived lymphoma. To overcome the limitations of CAR-T, alternative immune cell therapies, including CAR-NK 
and CAR-macrophages, were developed $(1,22)$. Instead of CARmacrophages, we developed genetically engineered macrophages that secrete anti-EGFR scFv-Fc. Compared to CAR, which is fixed on the cell surface, the secreting of scFv-Fc provides a more potent therapeutic advantage in attacking tumors, because of an increased capability for movement and penetration. Because scFv-Fc can efficiently coat the tumor cells, the opsonized tumor cells should be effectively eliminated through ADCP by macrophages.

Many previous attempts to replace viral vectors for the genetic engineering of immune cells have not been successful (23). In this study, we successfully used CpG-free plasmid microporation for the efficient delivery of genes into primary BMDMs as well as RAW 264.7 macrophages. The commercially available CpGfree plasmid was engineered for size reduction by removing the S/MAR and $\beta$-globin MAR sequences. Although MARs have often been used in various vector systems to prolong transgene expression $(24,25)$, they have been reported to work in a context-dependent manner $(26,27)$. In our study, the removal of the two MARs increased the transfection efficiency, as well as the cell viability, without provoking any harmful effects on the sustainability of transgene expression. Our results indicate that the strategy used to reduce the plasmid size was better than the one used to insert beneficial cis-regulatory elements.

The highest transfection efficiency $(\sim 50 \%)$ was obtained in the primary BMDMs, with reasonably good cell viability $(\sim 60 \%)$, using pCGfd-GFP under the optimal microporation conditions. This result is a great improvement compared to previous reports (28). Plasmid transfection in BMDMs is a great challenge, because BMDMs can degrade nucleic acids, including DNA, into lysosomes via phagocytosis (29). The conventional methods (liposome, DEAD-dextran, and calcium phosphate co-precipitation) have previously failed to accomplish the efficient delivery of plasmids into BMDMs (30). Electroporation has been suggested as an alternative solution for BMDM transfection, because it can directly deliver plasmids; however, high levels of cell death ( $\sim 90 \%$ ) have been associated with this method (28). Zhang et al. reported that nucleofection achieved high transfection efficiency in BMDMs. They did not, however, analyze its cell viability (11). Our strategy improved both the transfection efficiency and cell viability, suggesting that unmethylated $\mathrm{CpG}$ sequences are the major cause of low transfection efficiency and high levels of cell death in BMDM transfections. As such, pCGfd vector microporation could be a promising tool for the genetic engineering of macrophages.

A non-viral method using the pCGfd vector was successfully applied for the generation of antibody-secreting macrophages. We selected the antibody form of scFv-Fc to minimize the transgene size and use the opsonization function of Fc. RAW 264.7 macrophages transfected by pCGfd-aEGFR-scFv-Fc were able to secrete anti-EGFR scFv-Fc, thereby efficiently phagocytizing tumor cells expressing EGFR. The experiment using EGFR ${ }^{\mathrm{KO}}$ A431 cells proved that the process of phagocytosis occurred via ADCP. Since anti-EGFR scFv-Fc-secreting macrophages were shown to be effective, it could be extended by replacing the anti-EGFR antibody with other monoclonal antibodies already approved for use in cancer therapies. In our proof-of-concept experiments, we used only RAW 264.7 macrophages, because the use of BMDMs, which require the sacrifice of live mice, was limited. However, similar results could be obtained, considering the reasonably good transfection efficiency of the BMDMs, which was achieved by using pCGfd vector-based microporation.

We confirmed that anti-EGFR scFv-Fc-secreting macrophages retarded the growth of $\mathrm{EGFR}^{+}$tumors in mice. Although the inhibition of tumor growth was visible in this in vivo experiment, it was somewhat disappointing that the anti-EGFR scFv-Fcsecreting macrophages could not fully eradicate the tumors. It was speculated that the antigen-presenting function of macrophages did not work in the nude mice of the xenograft experiment, which was unable to generate mature T cells. Macrophages can present phagocytosed peptide fragments to T cells, subsequently inducing an adaptive immune response against the tumors. However, anti-EGFR scFv-Fc-secreting macrophages can eliminate only EGFR ${ }^{+}$tumor cells via ADCP in nude mice, without the help of the adaptive immune response. If antibody-secreting macrophages were to be injected into immunocompetent mice, they might generate a much greater therapeutic effect via their antigen-presenting function, eliciting an adaptive immune response in addition to ADCP.

In summary, in this study, we developed a CpG-free plasmid microporation method for the efficient transfection of macrophages and proved that these engineered macrophages were able to secrete antibodies and eliminate tumors via ADCP. As such, the findings presented here will contribute to the development of novel immune cell therapies for the treatment of cancer.

\section{MATERIALS AND METHODS}

The detailed methods are described in the "Supplementary Materials and Methods".

\section{ACKNOWLEDGEMENTS}

This work was supported by the grants of the National Research Foundation of Korea [NRF-2018R1A2B6003237], NextGeneration BioGreen 21 Program of the Rural Development Administration [PJ013320], and Korea Research Institute of Bioscience and Biotechnology (KRIBB) Research Initiative Program.

\section{CONFLICTS OF INTEREST}

The authors have no conflicting interests.

\section{REFERENCES}

1. Leslie M (2018) New cancer-fighting cells enter trials. Science 361, 1056-1057

2. Overdijk MB, Verploegen S, Bogels M et al (2015) 
Antibody-mediated phagocytosis contributes to the anti-tumor activity of the therapeutic antibody daratumumab in lymphoma and multiple myeloma. MAbs 7, 311-321

3. Chao MP, Alizadeh AA, Tang C et al (2010) Anti-CD47 antibody synergizes with rituximab to promote phagocytosis and eradicate non-Hodgkin lymphoma. Cell 142, 699-713

4. Shi Y, Fan X, Deng H et al (2015) Trastuzumab triggers phagocytic killing of high HER2 cancer cells in vitro and in vivo by interaction with Fcgamma receptors on macrophages. J Immunol 194, 4379-4386

5. Kurdi AT, Glavey SV, Bezman NA et al (2018) AntibodyDependent Cellular Phagocytosis by Macrophages is a Novel Mechanism of Action of Elotuzumab. Mol Cancer Ther 17, 1454-1463

6. Michaud HA, Eliaou JF, Lafont V, Bonnefoy N and Gros L (2014) Tumor antigen-targeting monoclonal antibody-based immunotherapy: Orchestrating combined strategies for the development of long-term antitumor immunity. Oncoimmunology 3, e955684

7. Lee S, Kivimae S, Dolor A and Szoka FC (2016) Macrophage-based cell therapies: The long and winding road. J Control Release 240, 527-540

8. Genard G, Lucas S and Michiels C (2017) Reprogramming of Tumor-Associated Macrophages with Anticancer Therapies: Radiotherapy versus Chemo- and Immunotherapies. Front Immunol 8, 828

9. Engelman A (2005) The ups and downs of gene expression and retroviral DNA integration. Proc Natl Acad Sci U S A 102, 1275-1276

10. Shayakhmetov DM, Gaggar A, Ni S, Li ZY and Lieber A (2005) Adenovirus binding to blood factors results in liver cell infection and hepatotoxicity. J Virol 79, 7478-7491

11. Zhang X, Edwards JP and Mosser DM (2009) The expression of exogenous genes in macrophages: obstacles and opportunities. Methods Mol Biol 531, 123-143

12. Hardee $\mathrm{CL}$, Arevalo-Soliz LM, Hornstein BD and Zechiedrich L (2017) Advances in Non-Viral DNA Vectors for Gene Therapy. Genes (Basel) 8, 65

13. Mun JY, Shin KK, Kwon O, Lim YT and Oh DB (2016) Minicircle microporation-based non-viral gene delivery improved the targeting of mesenchymal stem cells to an injury site. Biomaterials 101, 310-320

14. Latz E, Schoenemeyer A, Visintin A et al (2004) TLR9 signals after translocating from the ER to CpG DNA in the lysosome. Nat Immunol 5, 190-198

15. Hyde SC, Pringle IA, Abdullah S et al (2008) CpG-free plasmids confer reduced inflammation and sustained pulmonary gene expression. Nat Biotechnol 26, 549-551

16. Lesina E, Dames P, Flemmer A et al (2010) CpG-free plasmid DNA prevents deterioration of pulmonary function in mice. Eur J Pharm Biopharm 74, 427-434

17. Lesina E, Dames P and Rudolph C (2010) The effect of
CpG motifs on gene expression and clearance kinetics of aerosol administered polyethylenimine (PEI)-plasmid DNA complexes in the lung. J Control Release 143, 243-250

18. Pringle IA, Hyde SC, Connolly MM et al (2012) CpG-free plasmid expression cassettes for cystic fibrosis gene therapy. Biomaterials 33, 6833-6842

19. Mann CJ, Anguela XM, Montane J et al (2012) Molecular signature of the immune and tissue response to non-coding plasmid DNA in skeletal muscle after electrotransfer. Gene Ther 19, 1177-1186

20. Yew NS, Zhao H, Przybylska M et al (2002) CpG-depleted plasmid DNA vectors with enhanced safety and long-term gene expression in vivo. Mol Ther 5, 731-738

21. Hodges BL, Taylor KM, Joseph MF, Bourgeois SA and Scheule RK (2004) Long-term transgene expression from plasmid DNA gene therapy vectors is negatively affected by CpG dinucleotides. Mol Ther 10, 269-278

22. Morrissey MA, Williamson AP, Steinbach AM et al (2018) Chimeric antigen receptors that trigger phagocytosis. eLife 7, e36688

23. Mosaad YM (2014) Hematopoietic stem cells: an overview. Transfus Apher Sci 51, 68-82

24. Piechaczek C, Fetzer C, Baiker A, Bode J and Lipps HJ (1999) A vector based on the SV40 origin of replication and chromosomal S/MARs replicates episomally in $\mathrm{CHO}$ cells. Nucleic Acids Res 27, 426-428

25. Ramezani A, Hawley TS and Hawley RG (2003) Performance- and safety-enhanced lentiviral vectors containing the human interferon-beta scaffold attachment region and the chicken beta-globin insulator. Blood 101, 4717-4724

26. Schubeler D, Mielke C, Maass K and Bode J (1996) Scaffold/matrix-attached regions act upon transcription in a context-dependent manner. Biochemistry 35, 11160-11169

27. Giannakopoulos A, Stavrou EF, Zarkadis I, Zoumbos N, Thrasher AJ and Athanassiadou A (2009) The functional role of S/MARs in episomal vectors as defined by the stressinduced destabilization profile of the vector sequences. J Mol Biol 387, 1239-1249

28. Stacey KJ, Ross IL and Hume DA (1993) Electroporation and DNA-dependent cell death in murine macrophages. Immunol Cell Biol 71 ( Pt 2), 75-85

29. Rupprecht AP and Coleman DL (1991) Transfection of adherent murine peritoneal macrophages with a reporter gene using DEAE-dextran. J Immunol Methods 144, 157-163

30. Thompson CD, Frazier-Jessen MR, Rawat R, Nordan RP and Brown RT (1999) Evaluation of methods for transient transfection of a murine macrophage cell line, RAW 264.7. Biotechniques 27, 824-826, 828-830, 832

31. Shin DM, Yang CS, Yuk JM et al (2008) Mycobacterium abscessus activates the macrophage innate immune response via a physical and functional interaction between TLR2 and dectin-1. Cell Microbiol 10, 1608-1621 